\title{
On Core Convection and the Geodynamo: Effects of high electrical and thermal conductivity
}

\author{
David Gubbins $^{\mathrm{a}, \mathrm{b}}$, Dario Alfè ${ }^{\mathrm{c}},{\text { Chris } \text { Davies }^{\mathrm{a}} \text {, Monica Pozzo }}^{\mathrm{c}}$ \\ ${ }^{a}$ School of Earth 85 Environment, University of Leeds, Leeds LS2 9JT, United Kingdom \\ ${ }^{b}$ Institute of Geophysics and Planetary Physics, University of California at San Diego, \\ Scripps Institution of Oceanography, 9500 Gilman Drive, La Jolla, CA 92093-0225, USA \\ ${ }^{c}$ Department of Earth Sciences, Department of Physics and Astronomy, London Centre \\ for Nanotechnology and Thomas Young Centre at UCL, University College London, \\ Gower Street, London WC1E 6BT, United Kingdom
}

\section{Abstract}

Recent theory and experiment suggest the thermal and electrical conductivities of the Earth's core are 2-4 times higher than previously thought. This has important consequences for the core's thermal history and behaviour of the geodynamo. The conductivities increase with depth, with a discontinuous jump at the inner core boundary caused by the change in composition and phase change to a solid. Properties of putative core alloys are now sufficiently well known to make it worth exploring the effects of their variation with depth within the core. The magnetic decay times are increased to $58 \mathrm{kyr}$ for the whole core, considerably longer than the advection time (the time it takes fluid to traverse the outer core), and 9 kyr for the solid inner core. Heat conducted down the adiabat through the core-mantle boundary is in excess of 15 TW, which is one third of the Earth's total heat loss and 2-3 times higher than most estimates. The core can be stirred by chemical convection against a stable thermal gradient, but at a cost. We estimate the minimum heat flux required to sustain thermal dissipation alone to be 5-8 TW, but 
this is almost certainly a gross underestimate because it leaves nothing for convective or dynamo processes. Conduction gradients for cooling rates corresonding to these minimum heat fluxes are subadiabatic in the top $740 \mathrm{~km}$ of the core, which is also unlikely because geomagnetic secular variation requires upwelling somewhere near the core surface. Lateral variations in heat flux at the core-mantle boundary could easily be large enough to exceed the adiabatic value in some places, leading to mixing throughout the upper core. This not only reduces the total heat flux required to produce a well-mixed core, but also explains how mantle anomalies can exert a strong influence on core convection and the form of the geomagnetic field at the core surface. We propose a model of core convection that is vigorous in the lower part and very weak in the upper part.

Keywords: Earth's core; Geodynamo

\section{Introduction}

Until recently calculations of the core's thermal history and power supply for the geodynamo have been limited by poor knowledge of the material properties of likely core materials. The last decade has seen great improvements in both theoretical and experimental determinations of the properties of iron and iron alloys at high temperature and pressure, including density, seismic parameter, melting temperature, Grüneisen's parameter, material diffusivities, specific and latent heats, viscosity, and chemical potential. Studies of mixtures have extended to silicon, sulphur, oxygen, carbon, and hydrogen (Poirier, 1994; Alfè et al., 2002; Badro et al., 2014). Although some uncertainty remains, there is a remarkable degree of agreement between many 
studies.

Most recently, the all-important thermal and electrical diffusivities of Fe-Si alloys have been measured experimentally (Gomi et al., 2013) and calculated theoretically (de Koker et al., 2012; Pozzo et al., 2012, 2013, 2014) at core pressures and temperatures; they are found to be some 2-7 times higher than the widely-used estimates of Stacey and Anderson (2001) and Stacey and Loper (2007) rather than lower, as thought by some previous authors [e.g. Davies (2007)]. The higher values arise from a saturation that occurs when the mean free path between electron scattering events becomes comparable to the inter-atomic distance (Gunnarsson et al., 2003); the resistivity no longer follows the linear increase with temperature predicted by the Bloch-Grüneisen law but falls away at high temperature, leading to an increase in conductivity [Wiesmann et al. (1977) and Pozzo and Alfè, this issue]. This saturation effect had not been taken into account in previous estimates of the conductivities.

These very high values of thermal $(k)$ and electrical $(\sigma)$ conductivity have dramatic effects on the thermal history of the core and theory of the geodynamo. High $k$ means an enormous amount of heat is conducted down the adiabat and is not available to drive the dynamo. High $\sigma$ extends the magnetic diffusion time of the geomagnetic field, the time it would take the field to decay in the absence of any motion.

Core properties are now well enough known to reduce the uncertainties in core thermal history calculations dramatically. Furthermore, ab initio calculations give the depth-variation of most of these quantities accurately enough to make it worth discussing the depth-dependence of buoyancy forcing and 
dynamo driving. In this paper we therefore revisit estimates of present-day core heat flux, stratification, and dynamo power, including depth variations. We use a core model, described in Section 2, with an Fe-Si-O composition that matches the densities of the inner and outer cores. The crucial parameter is the density jump at the inner core boundary (ICB), most recently determined from normal mode eigenfrequencies as $0.8 \pm 0.2 \mathrm{gm} / \mathrm{cc}$ (Masters and Gubbins, 2003). We use 3 compositions corresponding to 3 values of the jump, 0.6 (PREM), 0.8 and $1.0 \mathrm{gm} / \mathrm{cc}$.

We first calculate the heat conducted down the adiabat for each density jump and a lower bound on the core cooling rate and heat flux required to mix the entire liquid outer core by thermo-chemical convection. The lower bound is less than that conducted down the adiabat at the core-mantle boundary (CMB) because compositional buoyancy acts against thermal buoyancy in places, driving heat downwards. In Section 4 we solve for the density profiles arising from the various sources of buoyancy as a guide to the convective stability as a function of depth within the core. In Section 5 we examine the effect of the electrical conductivity by calculating the magnetic decay modes for depth-varying conductivities and the effects of depth-variation of all the parameters on convection. We finish with a discussion of possible stable regions and conclusions for the true state of convection in the core. The whole discussion is restricted to the present-day core.

\section{The Core Model}

We assume a Fe-Si-O core with compositions that fit the seismic density values with a variable inner core boundary density jump, using the results 
from Alfè et al. (2002) and Alfè et al. (2007). S and Si partition almost equally between the liquid and solid phases, while $\mathrm{O}$ remains almost entirely in the liquid. Only $\mathrm{Si}$ is used here since $\mathrm{S}$ behaves in a closely similar fashion (Alfè et al., 2000) - replacing Si with S should make little difference. The seismic density jump determines the $\mathrm{O}$ content of the liquid core while the $\mathrm{Si}$ content adjusts to preserve the density of the inner core at values somewhat lower than those of pure iron. The density profiles for the outer core were calculated as described in Pozzo et al. (2013) and for the inner core in Pozzo et al. (2014).

Impurities lower the melting point, in the case of the core by many hundreds of degrees below the melting point of pure iron. The temperature at the ICB therefore varies with concentration, being lower for higher concentrations of light elements and therefore higher density jumps at the ICB. The adiabatic temperature is calculated by the usual integral

$$
T_{a}(r)=T_{\mathrm{o}} \exp \left\{\int_{r}^{r_{\mathrm{o}}} \frac{g \gamma}{\phi}\right\} d r=T_{\mathrm{i}} \exp \left\{-\int_{r_{\mathrm{i}}}^{r} \frac{g \gamma}{\phi}\right\} d r
$$

which leads to the ratio

$$
\frac{T_{a}^{\prime}}{T_{a}}=-\frac{g \gamma}{\phi}
$$

where prime denotes differentiation with respect to radius, $r$. Acceleration due to gravity, $g$, and the seismic parameter, $\phi$, are well determined by seismology. The thermodynamic Grüneisen parameter, $\gamma$, has been found from first principles calculations to be close to 1.5 throughout the core. The main uncertainty in $T_{a}(r)$ is $T_{\mathrm{i}}$, the melting temperature at the ICB; its gradient is proportional to $T_{\mathrm{i}}$ and is therefore shallower for the lower ICB temperatures associated with larger ICB density jumps, which in turn require 
higher concentrations of the impurity that lowers the melting point. The adiabat decreases significantly with depth in the core because of the decrease in $g / \phi$.

Another useful formula follows from the time derivative of (1). The right hand side depends only on physical properties of the core, and while the temperature may change by as much as $10 \%$, the exponent changes by a much smaller amount, of order $\alpha_{T} \gamma T$ or less than $1 \%$. Differentiating with respect to time and ignoring any secular change in $g \gamma / \phi$ gives

$$
\frac{1}{T_{a}} \frac{d T_{a}}{d t}=\frac{1}{T_{\mathrm{o}}} \frac{d T_{\mathrm{o}}}{d t},
$$

which allows us to refer the cooling rate at any depth in the core to that at the CMB.

The thermal expansion coefficient is related to the Grüneisen parameter by its thermodynamic definition:

$$
\alpha_{T}=\frac{\gamma C_{p}}{\phi} .
$$

Both $\gamma$ and the specific heat $C_{p}$ vary little across the core so $\alpha_{T}$ varies inversely as the seismic parameter: it decreases with pressure. The effect is substantial but has so far not received much attention in the context of core convection. A decrease in $\alpha_{T}$ with depth means a decrease in thermal buoyancy deep in the core, and a corresponding decrease in fluid flow and magnetic induction.

Mathematical variables and their values are given in Table 1. Variables that are model dependent are given in Table 2. Thermal and electrical conductivities are shown as a function of pressure in Figure 1. Both increase with depth, the thermal by some $50 \%$, the electrical less so because of the 
rising temperature (the Wiedemann-Franz law predicts $k \propto \sigma T$ ). Both are substantially larger in the inner core because of the lower concentration of light elements and solidification. The differences between the models may be unimportant in view of the uncertainty of the detailed composition of the core, but the increase with depth is significant and will probably apply to any core composition.

Curves for density jump $1.0 \mathrm{gm} / \mathrm{cc}$ are higher than expected in both figures because they are based on a different core density profile. Curves 0.6 are based on the PREM density. Curves 0.8 are based on a modified profile derived by adding enough $\mathrm{O}$ to the outer core to produce the right density jump, which makes the whole core lighter. This is inaccurate because seismology determines the mass of the entire core better than the density in the inner core. Curves 1.0, which were computed later, used a density profile that matched a recent estimate of the mass of the whole core, which involved removing Si from both cores and adding $\mathrm{O}$ to the outer core. Curves 0.8 were not recalculated because of the very high computational cost involved; they also illustrate the effect of changing the density profile, which is substantially larger than the (internal) errors of the ab initio calculation. We expect a selfconsistent density profile to place the 0.8 curves mid way between 0.6 and 1.0. This is a caution for those computing thermal histories, which require estimates of core density in the distant past rather than those based on direct seismic estimates - the profiles should be internally consistent in some way.

Thermal expansion decreases with pressure by about 60\% (Figure 3), as much as the thermal conductivity. Thermal expansion depends only on the seismic data, Grüneisen's constant, and the specific heat [equation (4)]: 
it does not depend significantly on composition and we can therefore be confident that it decreases substantially with depth in the core. Effects of these variations in depth are examined in Section 4.

\section{Core Heat Flow}

The new thermal conductivity estimates mean a large amount of heat is lost by conduction down the adiabat. Values for our 3 models are given in Table 3. They decrease with increasing ICB density jumps because the higher concentration of light elements lowers the melting point, which lowers the adiabatic gradient, but not by much. They are all about $15 \mathrm{TW}$, a third of the total heat flux issuing from the Earth's surface. This is much higher than any previous value and is difficult, if not impossible, to reconcile with mantle or core evolution.

The actual heat flux crossing the CMB can be less than the adiabatic value if chemical convection carries heat downwards, as originally suggested by Loper (1978), but a price has to be paid. Some cooling is essential because compositional convection is driven ultimately by freezing of outer core liquid at the bottom, which requires some heat loss from the CMB. The correct

balance can be calculated from the entropy equation, for example the one from Gubbins et al. (2004) equation (28):

$$
E_{k}+E_{\Phi}+E_{\alpha}=E_{R}+E_{S}+E_{L}+E_{P}+E_{H}+E_{g} .
$$

The left hand side has the dissipation entropy from heat, electric currents, and molecular diffusion (all of which are positive); the right hand side has the entropy contributions from various heat sources (chemical reaction, cooling, 


\begin{tabular}{lll}
\hline$c$ & concentration of light material & $\mathrm{m}$ \\
$T_{\mathrm{a}}$ & adiabatic temperature & $\mathrm{K}$ \\
$T_{\mathrm{co}}$ & cotemperature & $\mathrm{K}$ \\
$\partial T_{m} / \partial P$ & Melting gradient at ICB & $9.0 \mathrm{KGPa}^{-1}$ \\
$g$ & acceleration due to gravity & $\mathrm{ms}^{-2}$ \\
$q$ & heat source per unit volume (generic) & $\mathrm{Wm}^{-3}$ \\
$s$ & mass source per unit volume (generic) & $\mathrm{kgm}^{-3} \mathrm{~s}^{-1}$ \\
\hline$r_{\mathrm{i}}$ & inner core radius & $1.221 \times 10^{6} \mathrm{~m}$ \\
$r_{\mathrm{o}}$ & outer core radius & $3.485 \times 10^{6} \mathrm{~m}$ \\
$V_{\mathrm{oc}}$ & volume of outer core & $1.70 \times 10^{20} \mathrm{~m}^{3}$ \\
$M_{\mathrm{oc}}$ & mass of outer core & $1.85 \times 10^{24} \mathrm{~kg}$ \\
$M_{\mathrm{c}}$ & mass of whole core & $1.9477 \times 10^{24} \mathrm{~kg}$ \\
\hline$C_{P}$ & specific heat at constant pressure & $715 \mathrm{Jkg}^{-1} \mathrm{~K}^{-1}$ \\
$L$ & latent heat of outer core liquid & $0.75 \times 10^{6} \mathrm{Jkg}^{-1}$ \\
$\alpha_{c_{0}}$ & compositional expansion coefficient of oxygen & 1.10 \\
$\gamma$ & Grüneisen's constant & 1.5 \\
$\bar{\eta}_{\mathrm{c}}$ & Volume-averaged magnetic diffusivity, whole core & $0.6746 \mathrm{~m}^{2} \mathrm{~s}^{-1}$ \\
$\bar{\eta}_{\mathrm{i}}$ & Volume-averaged magnetic diffusivity, inner core & $0.5219 \mathrm{~m}^{2} \mathrm{~s}^{-1}$ \\
of radius and & inner core density jump. Ranges are from bottom to top of the core. \\
\hline & & are independent \\
\hline
\end{tabular}



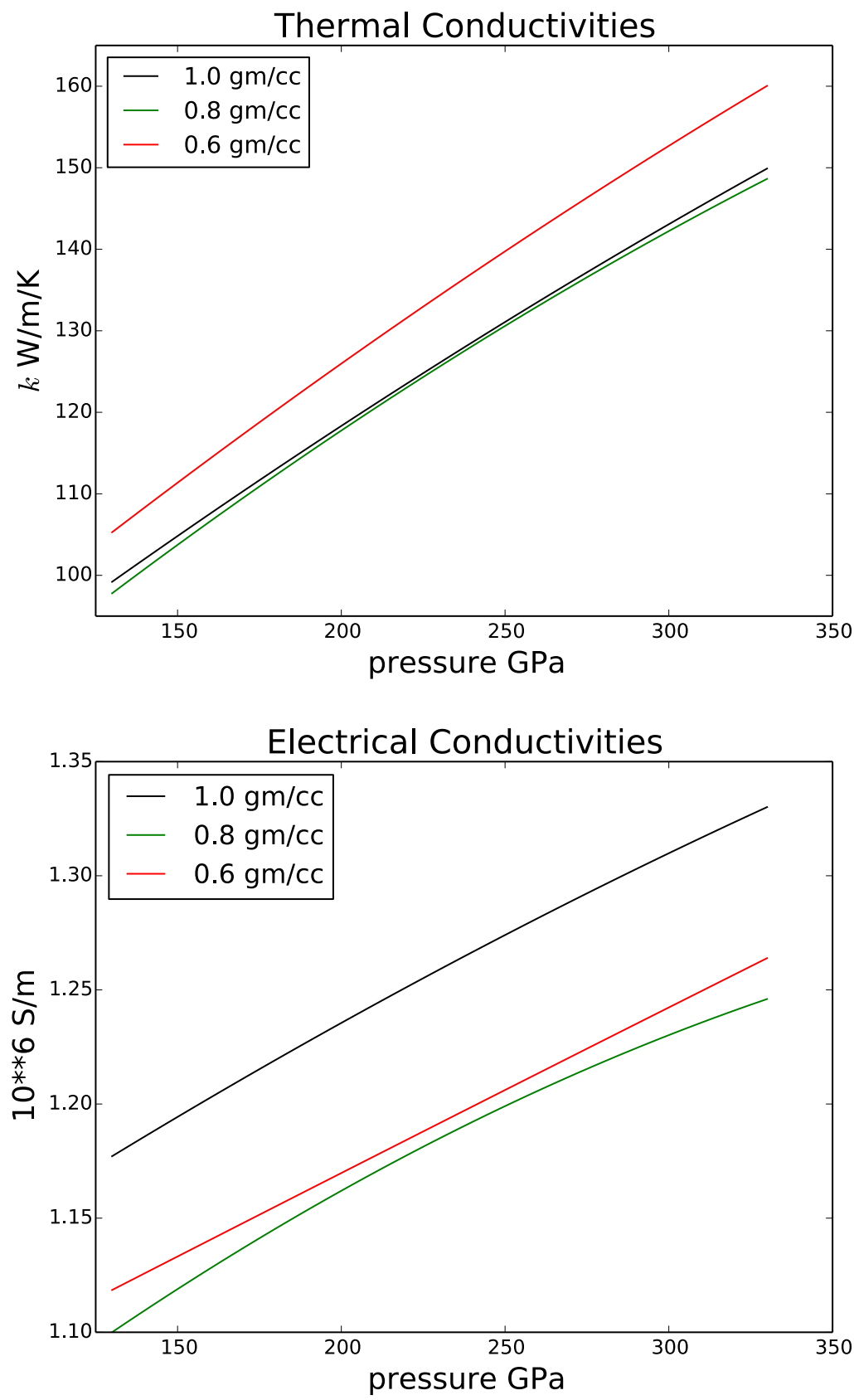

Figure 1: Thermal and electrical conductivity as a function of pressure in the outer core. Differences in the 3 plots are caused by different core temperatures caused by different melting temperatures at the ICB for different compositions and density jumps. 


\begin{tabular}{|l|lll|lll|lll|}
\hline$\Delta \rho$ & $T_{m}$ & $\mathrm{O}$ & $\mathrm{Si}$ & $k_{0}$ & $k_{1}$ & $k_{2}\left(10^{-4}\right)$ & $\sigma_{0}$ & $\sigma_{1}\left(10^{-4}\right)$ & $\sigma_{2}\left(10^{-8}\right)$ \\
\hline 0.598 & 5700 & 0.08 & 0.10 & 62.51 & 0.3508 & -1.674 & 1.022 & 7.454 & -4.028 \\
0.8 & 5500 & 0.13 & 0.08 & 54.51 & 0.3640 & -2.387 & 0.9539 & 1.280 & -119.6 \\
1.0 & 5300 & 0.17 & 0.02 & 59.75 & 0.3232 & -1.5197 & 1.055 & 10.11 & -53.46 \\
\hline
\end{tabular}

Table 2: Model-dependent quantities. Each model is defined by the density jump $(\Delta \rho \mathrm{gm} / \mathrm{cc})$ at the ICB. The first model has the density jump of PREM. $T_{m}$ is the melting temperature of the mixture at the ICB. Concentrations are mole fractions. The three coefficients for each of the conductivities are for a quadratic fit to calculations at 6 different pressures in GPa, viz. $k=k_{0}+k_{1} P+k_{2} P^{2}$. The conductivities of the inner core were taken to be linear fits betwen the values at the ICB and centre of the Earth, from Pozzo et al. (2014).

latent heat, pressure changes, radioactivity) and changes in gravitational energy consequent on separation of different chemical species. The heat terms all contain a thermodynamic efficiency factor involving the temperature at which heat is put into the system and the temperature at which it is taken out.

Previous studies have found $E_{k}$,

$$
E_{k}=\int k\left(\frac{\nabla T}{T}\right)^{2} d V
$$

to be the largest term on the left hand side because of conduction down the adiabat. The Ohmic term

$$
E_{\phi}=\int\left(\frac{\boldsymbol{J}^{2}}{\sigma T}\right) d V ; \quad \boldsymbol{J}=\frac{\nabla \times \boldsymbol{B}}{\mu_{0}}
$$

is essential if a dynamo is to operate. It is difficult to estimate because the larger contributions come from small scale magnetic fields internal to the core that are not observed, but dynamo simulations suggest it is no larger 
than $E_{k}$. Other contributions, from temperature fluctuations associated with the convection, mass diffusion, and viscosity, are usually neglected but this cannot be justified rigorously because they will be dominated by small scale turbulence. Higher thermal and electrical conductivities makes $E_{k}$ larger and $E_{\Phi}$ smaller. We therefore obtain a lower bound on the dissipation by ignoring everything except conduction down the adiabat.

The dominant terms on the right hand side come from cooling and gravitation, all of which are proportional to the cooling rate at the CMB. We ignore heat of reaction $\left(E_{R}\right)$ because it is small. $E_{P}$ is very small. $E_{H}$ could be large, depending only on the amount of radiogenic elements present. It is an inefficient way to produce entropy because the heat sources are uniformly distributed throughout the outer core: heat input is made at a similar temperature to heat output. Latent heat is more efficient because heat is added at the highest temperature and removed at the lowest. Chemical convection requires no efficiency factor as it stirs the core directly:

$$
E_{g}=\frac{Q_{g}}{T}
$$

where $Q_{g}$ is the rate of change of gravitational energy resulting from differentiation of light elements at the ICB. With these approximations, equation (5) reduces to

$$
E_{k}=E_{s}+E_{L}+E_{g}=\left(\hat{E}_{s}+\hat{E}_{L}+\hat{E}_{g}\right) \frac{D T_{\mathrm{o}}}{D t}
$$

where the quantities $\hat{E}$ are integrals of core properties. This equation can be used to find a lower bound on the cooling rate at the $\mathrm{CMB}$ required to maintain a well-mixed, adiabatic state throughout the outer core. 


\begin{tabular}{|l|l|l|l|l|l|l|l|}
\hline MODEL & $Q_{\mathrm{ad}}$ & $Q_{\text {min }}$ & $Q_{L}$ & $Q_{s}$ & $Q_{g}$ & $d T_{\mathrm{o}} d t$ & $E_{k}$ \\
\hline \hline CORE5700 & 16.81 & 7.71 & 3.42 & 3.13 & 1.16 & 61 & 6.02 \\
\hline CORE5500 & 15.08 & 5.97 & 2.32 & 2.33 & 1.32 & 45 & 5.62 \\
\hline CORE5300 & 14.73 & 5.36 & 1.90 & 2.06 & 1.40 & 40 & 5.66 \\
\hline
\end{tabular}

Table 3: Results of the calculation for the 3 models. $Q_{a d}$ is the heat conducted down the adiabat at the CMB, $Q_{\min }$ the sum of the heat sources, the remaining $Q$ 's are the individual contributions to $Q_{m i n}$, all in TW. The cooling rate corresponding to the minimum heat flux is $d T_{\mathrm{o}} / d t$, in $\mathrm{K} / \mathrm{Gyr}$ and $E_{k}$ is in units of $10^{8} \mathrm{~W} / \mathrm{K}$.

Given a cooling rate at the CMB we can sum the energy sources in the core and, by conservation of energy, calculate the heat flux crossing the CMB in quasi-steady state:

$$
Q_{\min }=\left(\hat{Q}_{s}+\hat{Q}_{L}+\hat{Q}_{g}\right) \frac{D T_{\mathrm{o}}}{D t}
$$

There is no reason for $Q_{\min }$ to be greater than the heat conducted down the adiabat and in fact it is not: the discrepancy is convected downwards by chemical convection. Further details are in Gubbins et al. (2004).

Results are given in Table 3. In all cases the minimum heat flux across the CMB is 2-3 times less than the heat flux down the adiabat. Note that these are lower bounds on the heat flux, not estimates or even realisable values. Other dissipations will be significant and a realistic estimate of core heat flux will be higher.

\section{Buoyancy Profiles}

We now determine whether basic state conduction profiles corresponding to the minimum heat flux models lead to a stable or an unstable density 
gradient. We achieve this in 3 stages: 1) establish the sources of buoyancy and heat flux in the Earth's core, 2) translate this into equivalent heat sources and boundary conditions for a thermally-driven, Boussinesq geodynamo model and 3) convert these heat sources and boundary conditions to basic state equivalent temperature profiles.

The Boussinesq equations apply to departures from a well-mixed, adiabatic basic state, which must be subtracted from the basic conducting state. The adiabat therefore acts as a stabilising temperature gradient, or equivalent heat sink, in a Boussinesq calculation. As in all solutions to the conduction equation, it is possible to derive equivalent heat sources and sinks and avoid the need to subtract an initial temperature profile. Conduction down the adiabat produces a heat deficit, removing heat that is not available to drive convection. It enters the basic state conduction equation as a heat sink with zero-flux upper and lower boundaries: The equivalent heat sink is simply

$$
q_{a}=\frac{1}{r^{2}} \frac{d}{d r}\left[r^{2} k(r) T_{\mathrm{a}}^{\prime}\right]
$$

For example, if $T_{\mathrm{a}}$ varies quadratically with $r$, and $k$ is independent of $r$, the equivalent heat sink is uniform.

The main sources of buoyancy in the outer core are latent heat, composition, and specific heat of cooling. Latent heat has no internal heat source; heat originates at the lower boundary (ICB) and, in steady state, the same amount of heat passes out through the upper boundary (CMB). In the heat conduction equation everything is specified by fixed temperature on the lower boundary and fixed heat flux on the upper boundary. The lower boundary condition simply determines the basic temperature level. The solution to the conduction equation in spherical geometry has the form $A+B / r$ : the 
gradient decreasing quadratically with radius. If this conduction solution is used as the basic state, the Boussinesq equations can be solved for departures from it with homogeneous boundary conditions.

Cooling provides a volumetric heat source equal to

$$
q_{\mathrm{s}}=-\rho C_{p} \frac{T_{\mathrm{a}}(r)}{T_{\mathrm{o}}} \frac{d T_{\mathrm{o}}}{d t}
$$

(Davies and Gubbins, 2011).

Compositional buoyancy is also provided through the lower boundary but the flux at the outer boundary is zero (ignoring barodiffusion and the possibility of influx from the mantle). The light element is well mixed throughout the outer core, causing a slow increase in the concentration there, the effects of which can be safely ignored. Subtracting out this slow increase leaves an equivalent, homogeneous, compositional sink. The appropriate conditions are fixed composition at the bottom boundary, zero flux at the upper boundary, and a uniform compositional sink in the fluid. The size of the mass sink is found by dividing the ICB flux by the mass of the outer core.

Compositional buoyancy enters the momentum equation in the form $\rho \alpha_{c} c$, where $c$ is concentration of the light component by mass while thermal buoyancy enters it in the form $\rho \alpha_{T} T$; composition can therefore be converted to an equivalent "cotemperature" by multiplying by the ratio of expansion coefficients $\alpha_{c} / \alpha_{T}$. The equivalent heat sink is found by multiplying the rate of fall of concentration with time by $\alpha_{c} / \alpha_{T}$ to convert it to an equivalent rate of drop of temperature and by $\rho C_{p}$ to convert it to a heat source per unit mass, leaving

$$
q_{c}=-\rho C_{p} \frac{\alpha_{c}}{\alpha_{T}} \frac{4 \pi r_{\mathrm{i}}^{2} \rho_{\mathrm{i}} c}{\tau_{i} M_{o c}} \frac{T_{\mathrm{i}}}{T_{\mathrm{o}}} \frac{d T_{\mathrm{o}}}{d t}
$$


where $c$ is the concentration and $\tau_{i}$ is the difference between the melting and adiabatic gradients at the ICB:

$$
\tau_{i}=\left(\frac{\partial T_{m}}{\partial r}-\frac{\partial T_{\mathrm{a}}}{\partial r}\right)_{\mathrm{ICB}}=-\rho_{i} g_{i} \frac{\partial T_{m}}{\partial P}-T_{\mathrm{a}}^{\prime}
$$

Basic state temperature gradients are found from the heat sources by solving the heat diffusion equation in steady state

$$
\frac{1}{r^{2}} \frac{d}{d r}\left[r^{2} k(r) T^{\prime}(r)\right]=-q
$$

with appropriate boundary conditions, constant cotemperature at the bottom and constant heat flux at the top. The solution is

$$
k(r) T^{\prime}(r)=-\frac{1}{r^{2}} \int_{0}^{r} x^{2} q(x) d x+A r
$$

where the constant $A$ must be determined by the upper boundary condition. The absolute value of the temperature is determined by the lower boundary condition, but it is not relevant to the solution for $T^{\prime}$.

Consider the 4 equivalent heat sources or sinks separately. It is best to use equivalent local heat fluxes $\left(k T^{\prime}\right)$ rather than equivalent temperature gradients because the adiabatic heat deficit involves the molecular conductivity whereas the other terms do not; in any subsequent dynamo calculation the molecular thermal conductivity is replaced by a the turbulent one.

Latent heat gives simply

$$
k T_{L}^{\prime}=\frac{\rho_{i} L}{\tau_{i}} \frac{T_{\mathrm{i}}}{T_{\mathrm{o}}} \frac{r_{\mathrm{i}}^{2}}{r^{2}} \frac{d T_{\mathrm{o}}}{d t} .
$$

Compositional convection gives the equivalent heat flux

$$
k T_{c}^{\prime}=-\frac{\rho C_{p} \alpha_{c}}{3 \alpha_{T}} C_{c} C_{r}\left(r-\frac{r_{\mathrm{o}}^{3}}{r^{2}}\right) \frac{d T_{\mathrm{o}}}{d t},
$$


where $C_{r}$ relates the rate of increase of inner core radius to the cooling rate

$$
\frac{d r_{\mathrm{i}}}{d t}=C_{r} \frac{d T_{\mathrm{o}}}{d t} ; \quad C_{r}=\frac{T_{\mathrm{i}}}{\tau_{i} T_{\mathrm{o}}}
$$

and $C_{c}$ relates the rate of release of light material at the ICB to the rate of advance of the boundary

$$
\frac{d c}{d t}=C_{c} \frac{d r_{\mathrm{i}}}{d t} ; \quad C_{c}=\frac{4 \pi r_{\mathrm{i}}^{2} \rho_{i} c}{M_{o c}} \frac{d r_{\mathrm{i}}}{d t}
$$

Specific heat requires the integral in (16):

$$
k T_{s}^{\prime}=\frac{C_{p}}{T_{\mathrm{o}}} \int_{0}^{r} \rho\left(r^{\prime}\right) T_{\mathrm{a}}\left(r^{\prime}\right) r^{\prime 2} d r^{\prime} \frac{d T_{\mathrm{o}}}{d t}=\frac{C_{p} I_{s}}{T_{\mathrm{o}}} \frac{d T_{\mathrm{o}}}{d t} .
$$

The adiabatic heat flux is just $k T_{\mathrm{a}}^{\prime}$.

The gravitational energy, $Q_{g}$, appearing in equation (10), arises from redistribution of mass within the core. It is converted to heat by dissipation associated with convection in the core, mainly magnetic. It does not appear as a heat source in the Boussinesq approximation and does not exist in the basic state, which is at rest. It does, however, represent a heat source in the convecting system and will affect the density gradient and thickness of any stable layer that develops near the CMB. Its distribution throughout the core depends on the convection and magnetic field generation, and is therefore unknown until the convection problem is solved, but its contribution to the heat loss at the CMB must equal $Q_{g}$ if equation (10) is to be satisfied. The corresponding heat flux there is $Q_{g} / 4 \pi r_{\mathrm{o}}^{2}$ and the temperature gradient a destabilising $-Q_{g} / 4 \pi r_{\mathrm{o}}^{2} k$. We include the term because our main interest is the stability of the density gradient near the CMB. It is rather small but possibly significant for convection driven primarily by composition, when the ohmic heating could form a large part of the total heat flux. We arbitrarily 
assume the heating to be uniform throughout the core, which is unlikely to produce serious error near the CMB where the heat flux is fixed.

Results are given in Figure 2 (a) for the $0.8 \mathrm{gm} / \mathrm{cc}$ density jump. The upper $740 \mathrm{~km}$ of the core is stratified. The change of inner core density jump makes very little difference to this thickness; it does make a difference to the adiabatic heat flux because, for a larger jump, enhanced compositional buoyancy compensates for the smaller thermal buoyancy resulting from a lower cooling rate.

Increasing the cooling rate from the minimum value (45 K/Gyr in Table 2) decreases the thickness of the stable layer; it disappears when the cooling rate reaches the point where the CMB heat flux equals that conducted down the adiabat (114 K/Gyr in this model). Another interesting case is when the core is slightly subadiabatic and the stable layer is thin. Figure 2(b) gives the results for a cooling rate $104 \mathrm{~K} / \mathrm{Gyr}$. The total heat flux is $13.7 \mathrm{TW}$ (compared with 15.1 down the adiabat) and $E_{k}=12.9 \mathrm{MW} / \mathrm{K}$. The stable layer is about $100 \mathrm{~km}$ thick. Note the larger contribution of the dissipative heating in this case.

There is an inconsistency in these calculations because $E_{k}$ was found assuming an adiabatic temperature gradient but the final result has a lower conducting gradient in the stable region; $E_{k}$ is therefore reduced. The difference is rather small: recalculating for the $740 \mathrm{~km}$ thick layer in Figure $2 \mathrm{a}$ decreases $E_{k}$ by $85 \mathrm{MWK}^{-1}$, or $13 \%$. A stable layer also decreases $Q_{g}$ because the light elements are now only mixed into the convection part of the core. $Q_{g} / T$ contributes substantially to the entropy budget because of its high efficiency. The cooling rate, increased by the smaller $Q_{g} / T$, then mul- 


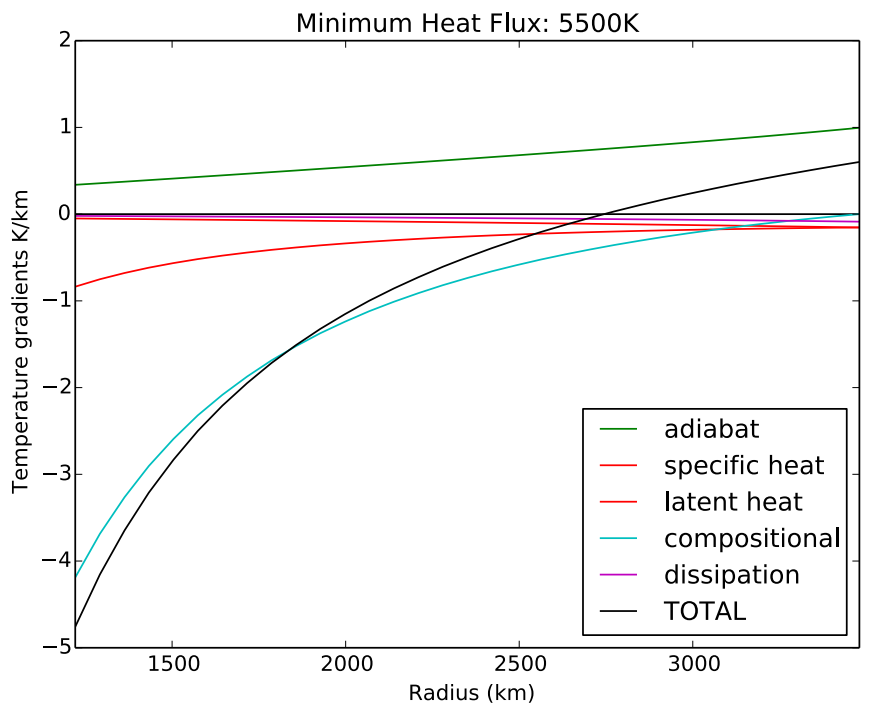

(a) $0.8 \mathrm{gm} / \mathrm{cc}, 45 \mathrm{~K} / \mathrm{Gyr}$

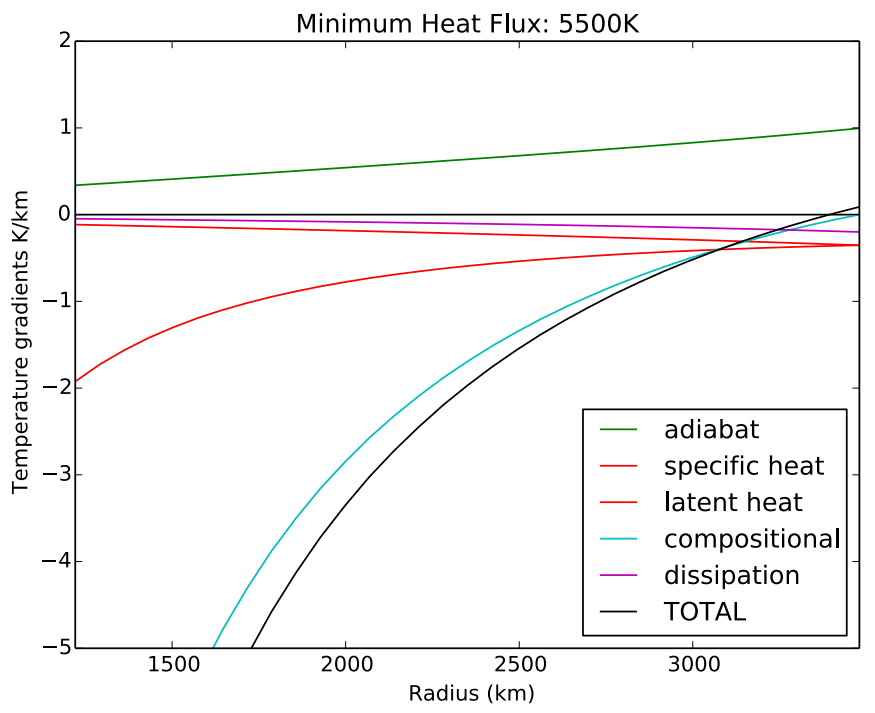

(b) $0.8 \mathrm{gm} / \mathrm{cc}, 104 \mathrm{~K} / \mathrm{Gyr}$

Figure 2: (a) Temperature gradients of a basic state including most plausible sources of buoyancy. Cooling and heat flow parameters as in Table 1. (b) As (a) but with a higher cooling rate, giving a stable layer $100 \mathrm{~km}$ thick. 
tiplies the much larger thermal terms in the energy equation. We could also improve the bound by including a lower bound on the dissipation entropy. All the gravitational energy is turned into heat via dissipation, mostly Ohmic; a lower bound on the associated entropy is therefore $Q_{g} / T_{\mathrm{i}}$ because $T_{\mathrm{i}}$ is the highest temperature in the system: $Q_{g} / T_{\mathrm{i}}=240 \mathrm{MWK}^{-1}$ for the $740 \mathrm{~km}$ model, larger than the $85 \mathrm{MWK}^{-1}$ decrease caused by the subadiabatic temperature. The estimate of minimum heat flux is therefore a conservative lower bound.

A stable conduction gradient does not imply stability when part of the system is convecting. Vigorous convection establishes a temperature profile close to adiabatic and transmits additional heat radially. Convection may penetrate into conductively-stable zones, and in a rotating system may create convection in a completely different region ["teleconvection", (Zhang and Schubert, 2000)]. In the core, conduction and adiabatic gradients are many orders of magnitude steeper than anything that can arise from the dynamics of convection (a rough back-of-the-envelope calculation gives a factor $10^{7}$ ). This implies very high Rayleigh numbers in unstable regions and extreme stratification that would suppress all radial motion, including penetrativeand tele-convection, in stable regions. The buoyancy period corresponding to these conduction gradients is of the order of a day, a regime in which Zhang and Schubert (2000) show that teleconvection would not occur. Weak convection gradients also mean the core cannot withstand substantial horizontal gradients and must remain well-mixed laterally even in stably stratified regions with no vertical motion.

A stable region is one in which the density gradient is steeper than the 
adiabatic gradient, i.e. a parcel of fluid displaced vertically that retains its heat and composition will experience a buoyancy force tending to return it to its original position. We take the stability boundary to be where the adiabatic gradient is balanced by the unstable density gradients. Further details are in Davies and Gubbins (2011).

\section{Effect on Geomagnetism and the Geodynamo}

The electrical conductivity determines the importance of magnetic diffusion in the core and is usually measured by a diffusion time, $l^{2} / \eta$, where $l$ is a relevant length scale and $\eta=1 / \mu_{0} \sigma$ is the magnetic diffusivity. A number of different choices have been made for the length scale, typically the radius of the whole core, the depth of the outer core (commonly used in geodynamo simulations), and radius of the inner core when dealing with the inner core. To be consistent and precise, we use the decay time of the slowest decaying mode in the whole core, as this is typical of the change in dipole moment caused by diffusion. The slowest decaying mode in a uniform sphere of radius $R$ is $R^{2} / \pi^{2} \eta$; the geometrical factor $\pi^{2}$ reduces the time by an order of magnitude, which explains the large variation of times seen in the literature. The diffusion time in the inner core is more difficult to identify because of the boundary condition: insulating is clearly not correct, but a conducting exterior simply reduces it to the time for the whole core. We use the insulating condition to give a rough idea of the time it would take the field deep in the inner core to change in response to changes in the outer core.

For the last decade most authors have used the estimates derived by 
Stacey and Anderson (2001), $\sigma=5 \times 10^{5}$ and $6.25 \times 10^{5} \mathrm{Sm}^{-1}$ for the outer and inner core respectively, giving decay times $25 \mathrm{kyr}$ and $3 \mathrm{kyr}$. The new values vary from $\sigma=1.1-1.3 \times 10^{6}$ for the outer core (increasing with depth) and $\sigma=1.5 \times 10^{6} \mathrm{Sm}^{-1}$ for the inner core, over twice Stacey \& Anderson's estimate. The diffusivity used in this paper is shown as a function of depth in Figure 3. The corresponding decay time for the whole core is therefore doubled to around $50 \mathrm{kyr}$ and that for the inner core to 2.4 times longer at $9.2 \mathrm{kyr}$.

More accurate decay times can be found by solving the diffusion equation with the diffusivity varying with radius. Gubbins and Roberts (1987) give the equations for the modes and a table of decay times for low-order modes with constant diffusivity. The magnetic field satisfies the vector equation

$$
\frac{\partial \boldsymbol{B}}{\partial t}=-\nabla \times(\eta \nabla \times \boldsymbol{B}) .
$$

Forming the scalar product with $\boldsymbol{B}$ and integrating over the sphere shows that all solutions decay when $\eta>0$. Modes are proportional to exp $-(t / \tau)$, where $\tau$ is the decay time. If $\eta$ depends only on $r$ they separate into poloidal and toroidal modes varying as a spherical harmonic $Y_{l}^{m}(\theta, \phi)$. Expanding the magnetic field in vector spherical harmonics

$$
\boldsymbol{B}=\sum_{l, m, n}\left\{\nabla \times\left[t_{l, n}^{m}(r) Y_{l}^{m} \boldsymbol{r}\right]+\nabla \times \nabla \times\left[p_{l, n}^{m}(r) Y_{l}^{m} \boldsymbol{r}\right] \exp \left(-t / \tau_{l, n}\right),\right.
$$

where $n$ is an overtone number, the number of zero crossings of the radial function, leaves second order differential equations for $t_{l, n}^{m}(r)$ and $p_{l, n}^{m}(r)$ :

$$
\begin{aligned}
\frac{1}{\tau_{l, n}} \frac{\partial t_{l, n}^{m}}{\partial t} & =\frac{\partial}{\partial r}\left[\eta \frac{\partial}{\partial r}\left(r t_{l, n}^{m}\right)\right]-\eta \frac{[(l+1)}{r^{2}} t_{l, n}^{m} \\
\frac{1}{\tau_{l, n}} \frac{\partial p_{l, n}^{m}}{\partial t} & =\frac{\eta}{r} \frac{\partial^{2}}{\partial r^{2}}\left(r p_{l, n}^{m}\right)-\eta \frac{l(l+1)}{r^{2}} p_{l, n}^{m} .
\end{aligned}
$$



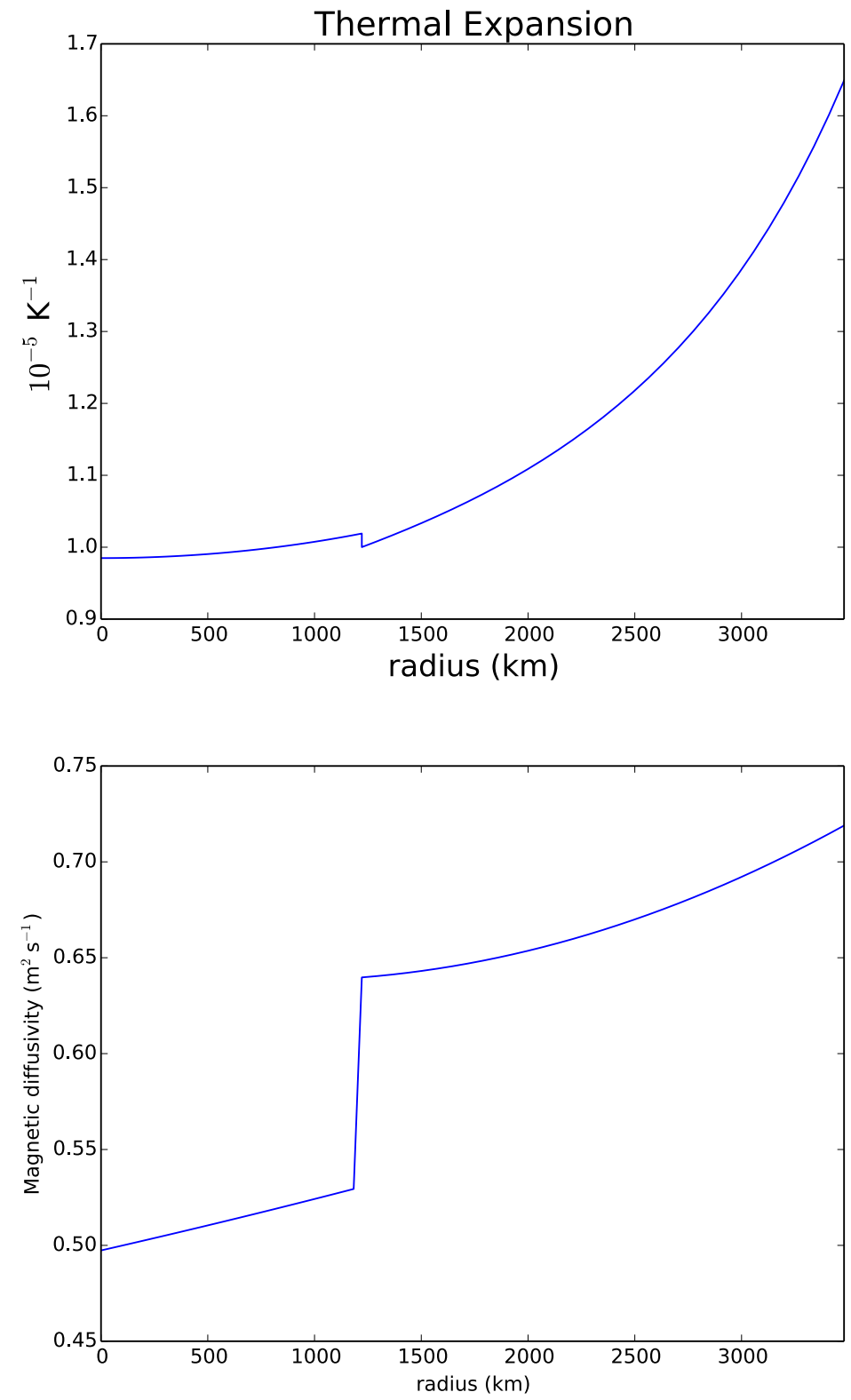

Figure 3: Thermal expansion coefficient as a function of pressure (upper) and magnetic diffusivity as a function of radius (lower). 


\begin{tabular}{|l|l|l|l|l|l|l|}
\hline$n=$ & 1 & 2 & 3 & 4 & 5 & 6 \\
\hline$l=1$ & $57.9(57.8)$ & $15.4(14.5)$ & $7.1(6.4)$ & $4.0(3.6)$ & $2.6(2.3)$ & $1.8(1.6)$ \\
$l=2$ & $28.1(28.3)$ & $9.9(9.6)$ & $5.2(4.8)$ & $3.2(2.9)$ & $2.1(1.9)$ & $1.5(1.4)$ \\
$l=3$ & $17.0(17.2)$ & $7.0(6.9)$ & $4.0(3.8)$ & $2.6(2.4)$ & $1.8(1.6)$ & $1.3(1.2)$ \\
$l=4$ & $11.5(11.5)$ & $5.35 .3)$ & $3.2(3.0)$ & $2.1(2.0)$ & $1.5(1.4)$ & $1.1(1.1)$ \\
$l=5$ & $8.4(8.5)$ & $4.2(4.2)$ & $2.6(2.5)$ & $1.8(1.7)$ & $1.3(1.2)$ & $1.0(0.9)$ \\
$l=6$ & $6.4(6.5)$ & $3.4(3.4)$ & $2.2(2.1)$ & $1.5(1.5)$ & $1.2(1.1)$ & $0.9(0.8)$ \\
\hline
\end{tabular}

Table 4: Poloidal decay times in kyr for the whole core for magnetic diffusivity varying with radius quadratically as $\eta=0.6464-0.0684 x+0.1413 x^{2}$, where $x$ is measured in core radii. Values in brackets are for a constant $\eta=0.6746 \mathrm{~m}^{2} \mathrm{~s}^{-1}$, the volumetric average of $\eta(x) . l$ denotes spherical harmonic degree and $n$ radial overtone number. The modes are degenerate in spherical harmonic order $m$.

The boundary conditions are $t(0)=p(0)=t\left(r_{\mathrm{o}}\right)=0$ and $p^{\prime}\left(r_{\mathrm{o}}\right)+(l+$ 1) $p\left(r_{\mathrm{o}}\right)=0$ (Gubbins and Roberts, 1987). The decay times are degenerate in $m$ and this superscript will be omitted henceforth. The toroidal equation (24) depends on the derivative $d \eta / d r$ but the poloidal equation does not because the associated electric currents are everywhere horizontal. For constant $\eta$ the solutions in radius are spherical Bessel functions. The toroidal modes are orthogonal with weight function $r^{2}$; the poloidal modes are not orthogonal because of the boundary condition. With variable $\eta(r)$ the toroidal modes remain orthogonal with the same weight function, $r^{2}$.

The decay times were computed using second order finite differences and solving the resulting algebraic eigenvalue problem. The results are in Table 5 . Values in parentheses are for a uniform diffusivity equal to the volume average over the whole core. As degree $l$ increases the eigenfunctions, like 


\begin{tabular}{|l|l|l|l|l|l|l|}
\hline$n=$ & 1 & 2 & 3 & 4 & 5 & 6 \\
\hline$l=1$ & $29.5(28.3)$ & $10.5(9.6)$ & $5.3(4.8)$ & $3.2(2.9)$ & $2.1(1.9)$ & $1.5(1.4)$ \\
$l=2$ & $17.6(17.2)$ & $7.3(6.9)$ & $4.1(3.8)$ & $2.6(2.4)$ & $1.8(1.6)$ & $1.3(1.2)$ \\
$l=3$ & $11.8(11.7)$ & $5.5(5.3)$ & $3.3(3.0)$ & $2.2(2.0)$ & $1.5(1.4)$ & $1.2(1.1)$ \\
$l=4$ & $8.6(8.5)$ & $4.3(4.2)$ & $2.7(2.5)$ & $1.9(1.7)$ & $1.3(1.2)$ & $1.0(0.9)$ \\
$l=5$ & $6.5(6.5)$ & $3.5(3.4)$ & $2.2(2.1)$ & $1.6(1.5)$ & $1.2(1.1)$ & $0.9(0.8)$ \\
$l=6$ & $5.2(5.2)$ & $2.9(2.8)$ & $1.9(1.8)$ & $1.4(1.3)$ & $1.1(1.0)$ & $0.8(0.8)$ \\
\hline
\end{tabular}

Table 5: As Table 5 for toroidal modes.

the spherical Bessel functions, concentrate towards the upper core and sample the higher diffusivity there, giving relatively longer decay times. High overtones sample the core at depth and tend to sample the lower diffusivity at depth. The higher modes are of interest because they represent the importance of diffusion of features with the length and time scales studied in secular variation. Solutions for yet higher modes can be estimated by asymptotic methods given in Gubbins and Roberts (1987)

The other important geomagnetic time scale, the advection time in the core $\left(r_{\mathrm{o}} / v\right)$ is independent of the diffusivity and so the magnetic Reynolds number (ratio of diffusion to advection times) is raised: the dynamo is driven harder and the frozen flux approximation used in determining core flow should be more accurate. Longer term phenomena such as polarity reversals appear very fast in the context of magnetic diffusion. The electrical conductivity increases with depth, diminishing the importance of diffusion there. Magnetic field changes in the inner core are controlled entirely by diffusion; the dipole decay time of 9.2 kyr may help stabilise the geodynamo 
and control the frequency of reversals once a dipole has been established (Gubbins, 1999).

The electrical conductivity increases with depth, causing the dynamo to be driven harder there. The adiabat is shallower at depth (Figure 2), reducing the heat deficit, but the thermal conductivity is larger, counteracting the effect somewhat. The thermal expansion coefficient decreases significantly with depth, also reducing the buoyancy. Compositional buoyancy is larger at depth and the composition expansion coefficient remains fairly constant. The dynamo is therefore driven mostly by compositional convection in the lower half of the core, while strong horizontal flow in the upper reaches of the core could still be a major contributor to generation of toroidal fields there. The picture emerges of a geodynamo operating in a highly turbulent convective regime in the lower half of the core, generating a magnetic field that is modified by upward continuation through a stable upper half, which is likely to enhance the large scale parts of the poloidal field at the core surface relative to the small scale parts. At the core surface the observed magnetic field will have larger scales than would be expected from dynamo models with vigorous convection in the upper reaches of the core.

\section{Discussion}

The very high core heat flux estimates that result from the new estimates of the thermal conductivity present serious difficulties for theories of mantle convection and the thermal evolution of the Earth. There are several options: 1) the core heat flux is indeed very high, around $15 \mathrm{TW}$, or one-third of the heat flux at Earth's surface; 2) there is a very thick, stable layer somewhere 
in the core; and 3) large lateral variations in CMB heat flux allow the top of the core to mix.

Option 1) is unpalatable for several reasons. First, it provides strong bottom-heating for mantle convection, which runs against some models (Davies, 2007). Secondly, the cooling rate must have been even higher in the past, making it difficult to explain magnetic field generation prior to inner core formation [Nimmo (2007), Davies, this issue]. Thirdly, if the core heat flux is sustained by radioactive heating, the proportion of radiogenic elements would have to be far higher than suggested hitherto [e.g. Murthy et al. (2003)].

Option 2) has some problems because it may be inconsistent with observations of geomagnetic secular variation if the stable layer is at the top of the core, as we predict here. Some core motion studies predict stability at the top of the core [e.g. Whaler (1980)] and the present flux expulsion seen on the core surface beneath the South Atlantic strongly suggests radial flow at a fairly shallow depth below the CMB (Gubbins, 1996, 2007). These studies are consistent with a thin stable layer, 50-100 km thick, but not with the $740 \mathrm{~km}$-thick layer resulting from modest CMB heat flux. There is also seismic evidence from seismology for a thin stratified layer at the top of the core (Eaton and Kendall, 2006; Helffrich and Kaneshima, 2010), but again only sampling a thin layer.

The stable layer may occur deeper down in the core, as suggested by Gomi et al. (2013). The exact location of the stable region depends critically on the depth dependence of the relevant parameters, notably thermal conductivity and expansion. Gomi et al. (2013) use constant $\alpha_{T}$ and an extrapolation of $k$ from the pressure of their experiment deep into the core. We use calculations 
for present-day seismic values and pressures that span the full range of the outer and inner cores. These give a strongly depth-varying $\alpha_{T}$ and a $k$ that agrees with experiment at lower pressure but is quite from Gomi et al.'s extrapolation to the deeper core.

Option 3) differs from option (2) only in the strength of the boundary heat flux anomalies. If the anomalies are much weaker than the subadiabatic heat flux the surface flow is likely to be decouple from the deeper convection; if they are comparable then the stable layer will be destroyed and replaced by a weakly convecting one. Suppose the maximum heat flux is 3 times an average of $6 / 4 \pi r_{\mathrm{o}}^{2} \mathrm{TW} / \mathrm{m}^{2}$, just above the minimum heat flux for model CORE5500 in Table 3, or $18 / 4 \pi r_{\mathrm{o}}^{2}$. This exceeds the adiabatic heat flux $\left(15 / 4 \pi r_{\mathrm{o}}^{2}\right)$ at that particular location. Core fluid would be unstable there, leading to lateral mixing to all other parts of the upper core because there is little or no resistance to horizontal flow. The core would be well mixed throughout, apart perhaps from a thin surface layer, despite having a mean stable density gradient when averaged laterally.

This scenario has much to recommend it.

It explains low secular variation in the Pacific, where the lowermost mantle is hot and stabilising. The coldest part of the mantle, and highest CMB heat flux, is likely to be beneath the general region of Indonesia, where two subduction zones have been active for long enough to have cooled the mantle all the way down to the core. Westward core flow starts here and generates the secular variation, along with flux expulsion, in the Atlantic hemisphere.

It enhances thermal core-mantle interaction because advection will be weak, 
which is known from dynamo simulations to promote the influence of boundary thermal anomalies on deeper convection (Sreenivasan and Gubbins, 2008). The time-averaged field appears to reflect the underlying convection: it is symmetrical about the equator, with concentrations of flux aligned with regions of likely downwelling flow (the four "main lobes") (Davies et al., 2009).

Vigorous convection is very likely to generate a complex magnetic field, not the dipole-dominated, spatially simple field observed at the core surface and seen in the current family of geodynamo simulations, which are necessarily limited to laminar flow, high Ekman number (high viscosity), low Rayleigh numbers (low applied compositional and thermal gradients), and Prandtl numbers close to unity. Confining the vigorous convection to the deeper part of the liquid core means the spatially complex fields will be attenuated in the very weakly convecting upper core, enhancing the dipole and reflecting the large scale thermal anomalies on the CMB.

The dynamics of such a stable region driven by strong boundary anomalies may be similar to those envisaged in Braginsky's "inner ocean" (Braginsky, 1999, 2006), for example by supporting magnetic Rossby waves (Braginsky, 1999; Buffett, 2014). The very strong stratification envisaged in option (2) will support short-period gravity waves but not typical MAC-waves that have a force balance that includes magnetic forces. Boundary forcing produces a buoyancy regime similar to that of penetrative convection in a weakly stratified zone. 


\section{Conclusions}

The high core thermal and electrical conductivities suggested by the most recent $a b$ initio calculations and experiments demand a rethink of the present and past thermal state of the core. A high core heat flux (8-15 TW) seems inevitable, implying a very young inner core $(<1 \mathrm{Ga})$. Inner core formation may well have occurred within the last few hundred million years, so it would be worth looking into the paleomagnetic record to see if there is any indication of a sudden boost to the power available to the geodynamo associated with addition of compositional driving. The style of core convection is vigorous at depth, driven mainly by compositional convection, weakening with radius as thermal convection takes over. Compositional buoyancy drives convection against a stable thermal gradient in mid-core, generating ohmic heating that could, in principle, be quite significant if compositional convection is strong and thermal buoyancy weak.

The heat deficit caused by conduction down the adiabat strengthens with radius; this combined with weaker compositional convection leads to an upper core that is stably stratified in the spherical average. It is worth noting that Gomi et al. (2013) derive a conduction profile that is unstable at the very top of the core, above a similar stable layer to ours (see also Labrosse, this isssue). This arises because their thermal conductivity increases more rapidly with depth. The depth dependence is based on an extrapolation from direct measurements with anchor points at $100 \mathrm{GPa}$ and room temperature, which are quite far from ccore conditions, especially the temperature. The direct measurements agree well with our ab initio calculations but the extrapolation diverges in the core. 
Geomagnetic secular variation suggests there must be upwelling close to the $\mathrm{CMB}$, within $100 \mathrm{~km}$ or less, vitiating the presence of a deep, unmixed upper core. The dilemma is resolved if lateral variations of heat flux around the CMB are great enough to overcome the thermal stratification in places. Those places are likely to be beneath the coldest parts of the lower mantle boundary layer, where heat transport across the CMB is highest. This scenario has much to recommend it: it could explain low secular variation in the Pacific and other consequences of strong mantle control on core convection. The observed field reflects mostly flow in the upper core and can therefore be larger scale and less turbulent than would be expected from the low Ekman number, high Rayleigh number, convection pertaining in the main dynamo region deeper down.

\section{Acknowledgements}

The paper has been much improved by constructive comments from 2 referees. DG is supported by NERC grant 1012052 "Rapid Dynamics in the Earth's Core", CD by a NERC Personal Fellowship HO1571X and a Green Foundation Postdoctoral Scholarship at IGPP, Scripps Institution of Oceanography. 


\section{References}

Alfè, D., Gillan, M.J., Price, G.D., 2002. Composition and temperature of the Earth's core constrained by combining ab initio calculations and seismic data. Earth Planet. Sci. Lett. 195, 91-98.

Alfè, D., Gillan, M.J., Price, G.D., 2007. Temperature and composition of the Earth's core. Contemporary Physics 48, 68-80.

Alfè, D., Price, G.D., Gillan, M.J., 2000. Constraints on the composition of the Earth's core from ab-initio calculations. Nature 405, 172-175.

Badro, J., Côté, A.S., Brodholt, J.P., 2014. A seismologically consistent compositional model of Earth's core. Proc. Nat. Acad. Sci. 111, 75427545 .

Braginsky, S., 2006. Formation of the stratified ocean of the core. Phys. Earth Planet. Int. 243, 650-656.

Braginsky, S.I., 1999. Dynamics of the stably stratified ocean at the top of the core. Phys. Earth Planet. Int. 111, 21-934.

Buffett, B., 2014. Geomagnetic fluctuations reveal stable stratification at the top of the Earth's core. Nature 507, 484-487.

Davies, C.J., Gubbins, D., 2011. A buoyancy profile for the Earth's core. Geophys. J. Int. 187, 549-563.

Davies, C.J., Gubbins, D., Jimack, P.K., 2009. Convection in a rapidly rotating spherical shell with an imposed laterally varying thermal boundary condition. J. Fluid Mech. 641, 335-358. 
Davies, G.F., 2007. Mantle regulation of core cooling: A geodynamo without core radioactivity? Phys. Earth. Planet. Int. 160, 215-229.

Eaton, D.W., Kendall, J.M., 2006. Improving seismic resolution of outermost core structure by multichannel analysis and deconvolution of broadband SmKS phases. Phys. Earth Planet. Int. 155, 104-109.

Gomi, H., Ohta, K., Hirose, K., Labrosse, S., Caracas, R., Verstraete, M.J., Hernlund, J.W., 2013. The high conductivity of iron and thermal evolution of the Earth's core. Phys. Earth Planet. Int. 224, 88-103.

Gubbins, D., 1996. A formalism for the inversion of geomagnetic data for core motions with diffusion. Phys. Earth Planet. Int. 98, 193-206.

Gubbins, D., 1999. The distinction between geomagnetic excursions and reversals. Geophys. J. Int. 137, F1-F3.

Gubbins, D., 2007. Geomagnetic constraints on stratification at the top of Earth's core. Earth Planets \& Space 59, 661-664.

Gubbins, D., Alfè, D., Masters, T.G., Price, D., 2004. Gross thermodynamics of 2-component core convection. Geophys. J. Int. 157, 1407-1414.

Gubbins, D., Roberts, P.H., 1987. Magnetohydrodynamics of the Earth's core. chapter 1, in: Jacobs, J.A. (Ed.), Geomagnetism Volume II. Academic Press, pp. 1-183.

Gunnarsson, O., Calandra, M., Han, J.E., 2003. Colloquium : Saturation of electrical resistivity. Rev. Mod. Phys. 75, 1085-1099. 
Helffrich, G., Kaneshima, S., 2010. Outer-core compositional stratification from observed core wave speed profiles. Nature 468, 807-809.

de Koker, N., Steinle-Neumann, G., Vlcek, V., 2012. Electrical resistivity and thermal conductivity of liquid Fe alloys at high $\mathrm{P}$ and $\mathrm{T}$, and heat flux in Earth's core. Proc. Natl. Acad. Sci. 109, 4070-4073.

Loper, D.E., 1978. Some thermal consequences of a gravitationally powered dynamo. J. Geophys. Res. 83, 5961-5970.

Masters, T.G., Gubbins, D., 2003. On the resolution of density within the Earth. Phys. Earth Planet. Int. 140, 159-167.

Murthy, V.M., van Westrenen, W., Fei, Y.W., 2003. Experimental evidence that potassium is a substantial radioactive heat source in planetary cores. Nature 423, 163-165.

Nimmo, F., 2007. Energetics of the core, in: Schubert, G. (Ed.), Treatise on Geophysics. Elsevier B. V.. volume 8. chapter 2, pp. 31-66.

Poirier, J.P., 1994. Light elements in the Earth's outer core - a critical review. Phys. Earth Planet. Inter. 85, 319-337.

Pozzo, M., Davies, C., Gubbins, D., Alfé, D., 2012. Thermal and electrical conductivity of iron at Earth's core conditions. Nature 485, 355-U99.

Pozzo, M., Davies, C., Gubbins, D., Alfé, D., 2013. Transport properties for liquid silicon-oxygen-iron mixtures at Earth's core conditions. Physical Review B 87, 101410. 
Pozzo, M., Davies, C., Gubbins, D., Alfé, D., 2014. Thermal and electrical conductivity of iron at Earth's core conditions. Earth Planet. Sci. Lett. 393, 159-164.

Sreenivasan, B., Gubbins, D., 2008. Dynamos with weakly convecting outer layers: implications for core-boundary locking. Geophys. Astrophys. Fluid Dyn. 102, 395-407.

Stacey, F.D., Anderson, O.L., 2001. Electrical and thermal conductivities of Fe-Ni-Si alloy under core conditions. Phys. Earth Planet. Int. 124, 153-162.

Stacey, F.D., Loper, D.E., 2007. A revised estimate of the conductivity of iron alloy at high pressure and implications for the core energy balance. Phys. Earth Planet. Int. 161, 13-18.

Whaler, K.A., 1980. Does the whole of the Earth's core convect? Nature $287,528-530$.

Wiesmann, H., Gurvitch, M., Lutz, H., Ghosh, A., Schwarz, B., Strongin, M., Allen, P.B., Halley, J.W., 1977. Simple model for characterizing the electrical resistivity in A-15 superconductors. Phys. Rev. Lett. 38, 782-785.

Zhang, K., Schubert, G., 2000. Teleconvection: Remotely Driven Thermal Convection in Rotating Stratified Spherical Layers. Science 290, 19441947. 